\title{
ESTUDIO DE LA EPIDERMIS FOLIAR EN ALGUNAS ESPECIES DE BURSERA JACQ. EX L. (BURSERACEAE) Y SUS IMPLICACIONES TAXONÓMICAS
}

\author{
Mercedes E. Castro ${ }^{1,2}$ y Pedro Torrecilla ${ }^{1}$ \\ ${ }^{1}$ Universidad Central de Venezuela, Facultad de Agronomía, Instituto \\ de Botánica Agrícola, Apdo. 4579, 2101 Maracay, Edo. Aragua, Venezuela. \\ 2Autor para la correspondencia: castrom@agr.ucv.ve, laportte@hotmail.com
}

\section{RESUMEN}

Se realizó el estudio de las características de la epidermis foliar de seis especies de Bursera. Las muestras fueron analizadas utilizando técnicas de microscopía óptica y electrónica de barrido (MEB). Todas presentaron hojas hipostomáticas, estomas anomocíticos y el mismo tipo de tricomas glandulares y tectores, con excepción de B. karsteniana que es glabra. La micromorfología de la epidermis foliar vista al MEB mostró que todas tienen paredes anticlinales rectas, sin embargo éstas presentan una combinación única de caracteres para cada especie. Los análisis fenéticos separan dos grupos, uno formado por los tres representantes del subgénero Bursera y otro por los tres pertenecientes al subgénero Elaphrium, lo cual apoya la mencionada subdivisión. Los caracteres de la epidermis foliar mostraron un alto valor taxonómico en Bursera.

Palabras clave: Bursera, características de epidermis foliar, taxonomía, Venezuela.

\begin{abstract}
Leaf epidermis characteristics of six species of Bursera were studied using light and scanning electron microscopy (SEM). All species possess hypostomatic leaves, anomocytic stomata, and similar glandular and eglandular trichomes, with the exception of B. karsteniana which is glabrous. SEM of the leaf epidermis revealed that all have straight anticlinal walls, but these have a suite of sculptural characters unique to each species. Phenetic analyses separate the species into two groups, one formed by the three representatives of subgenus Bursera and the other by the three representatives of
\end{abstract}


subgenus Elaphrium, supporting the aforementioned subdivision. Leaf epidermis features are taxonomically important for the genus.

Key words: Bursera, leaf epidermis characteristics, taxonomy, Venezuela.

\section{INTRODUCCIÓN}

El género Bursera Jacq. ex L. está conformado por aproximadamente 100 especies del Nuevo Mundo que se distribuyen desde el sur de Estados Unidos hasta Suramérica y el Caribe (Espinosa et al., 2006). Según Gillet (1980) está dividido en dos subgéneros (Bursera y Elaphrium), que se corresponden a las secciones Bursera y Bullockia propuestas por McVaugh y Rzedowski (1965), éstos se diferencian por las características de la corteza externa, la forma del raquis, la pubescencia de las flores y el número de valvas en el fruto (McVaugh y Rzedowski, 1965; Daly, 1993, 1997).

En Suramérica se ha señalado la presencia de siete especies (Daly, 1993); Bursera glabra (Jacq.) Triana \& Planch., B. karsteniana Engl. y B. inversa Daly son endémicas de Colombia y Venezuela, B. malacophylla B. L. Rob. es exclusiva de las islas Galápagos, mientras que $B$. graveolens (Kunth) Triana \& Planch., $B$. simaruba (L.) Sarg. y B. tomentosa (Jacq.) Triana \& Planch. son de más amplia distribución.

Los estudios taxonómicos en Bursera tienden a ser complejos, ya que este grupo está pobremente colectado y los especímenes de herbario en su mayoría presentan escasas flores y frutos. Debido a esto, la taxonomía del género se ha basado principalmente en las características morfológicas de la corteza externa, de la hoja y del fruto (Becerra y Venable, 1999).

Trabajos recientes basados en secuencias de ADN evidencian la naturaleza monofilética de Bursera y de los dos subgéneros que integran el conjunto (Becerra y Venable, 1999; Becerra, 2003), planteamiento que es apoyado por las características morfológicas de las plántulas (Andrés-Hernández y Espinosa-Organista, 2002). No obstante, Weeks et al. (2005), al realizar el estudio de la historia evolutiva de las Burseraceae, concluyen que Bursera es parafilético, ya que un grupo monofilético debería incluir a Commiphora. Sin embargo, Becerra et al. (2012) hacen una reconstrucción filogenética, basada en caracteres moleculares, de ambos géneros y concluyen que Bursera es un conjunto monofilético.

Tradicionalmente los caracteres reproductivos han sido de utilidad para separar las especies de plantas; no obstante, los vegetativos también pueden brindar información taxonómica valiosa. Stace $(1965,1980,1984)$ ha indicado que entre los 
órganos vegetativos para los estudios de orden sistemático, las características de las hojas son las más ampliamente utilizadas, mientras que Srivastava (1978) señala que la epidermis foliar es una de las fuentes de información más útil para la resolución de problemas de esta índole. Algunos rasgos de las hojas, tales como el aparato estomático, tipo de tricomas, forma de las células epidérmicas y la ornamentación de la cutícula tienen significancia taxonómica y sistemática en diferentes grupos de plantas (Barthlott, 1981; Barthlott et al., 1998; Loza-Cornejo y Terrazas, 2003; Dean y Ashton, 2008; Ogunkunle y Oladele, 2008; Yang et al., 2008; Zou et al., 2008).

Con base en lo anterior, nos planteamos evaluar la utilidad taxonómica y el posible valor filogenético de la epidermis foliar de seis especies de Bursera presentes en Venezuela, de los subgéneros Bursera (B. karsteniana, B. inversa, B. simaruba) y Elaphrium (B. glabra, B. graveolens, B. tomentosa).

\section{MATERIALES Y MÉTODOS}

\section{Material vegetal}

De las especies de Bursera estudiadas se colectaron hojas maduras en plena expansión foliar en poblaciones naturales de Venezuela y se tomaron muestras de herbario de tres individuos por especie, con excepción de $B$. inversa para la que se tuvo acceso a solo un árbol. El material fresco fue fijado inmediatamente en FAA 70\% (alcohol: ácido acético: formol en proporción 90:5:5), mientras que las muestras herborizadas se hidrataron en lactofenol por tiempos variables según las características de cada especie, y posteriormente fueron fijadas en FAA 70\%. Los especímenes de herbario utilizados en el estudio se presentan en el Apéndice.

\section{Microscopía óptica (MO)}

Para el estudio de la epidermis foliar, se tomó aproximadamente $1 \mathrm{~cm}^{2}$ de la porción media del tejido fijado y se colocó en cápsulas de Petri en una solución de hipoclorito de sodio $(5.25 \%)$ a temperatura ambiente hasta que ambas epidermis se separaron de los tejidos internos. Posteriormente el material fue teñido con una solución de azul de toluidina acuosa a $1 \%$ y se montaron láminas semi-permanentes en una solución de agua:glicerina (1:1) hasta su observación. Las fotografías se obtuvieron a partir de una cámara digital acoplada a un microscopio óptico marca Nikon y procesadas con un programa de análisis de imágenes. El índice estomático fue calculado siguiendo a Wilkinson (1979). 
Microscopía electrónica de barrido (MEB)

Se tomaron porciones de aproximadamente $1 \mathrm{~cm}^{2}$ de lámina foliar, las cuales se deshidrataron en una batería creciente de etanol $(30 \%, 50 \%, 70 \%, 95 \%$ y $100 \%)$ siguiendo lo propuesto por Shuff y Thomas (1993). Posteriormente se realizó el secado por punto crítico en un secador Bal-Tec CPD 030, para después cubrir el material con una capa fina de oro-paladio utilizando un cubridor iónico Baltec ScB 050. Las observaciones se hicieron en un microscopio electrónico de barrido marca Phillips XL-20 a 10Kv. La descripción de los caracteres micromorfológicos se hizo siguiendo la clasificación de Barthlott (1981) y Barthlott et al. (1998).

Análisis de datos

Se realizó un análisis fenético utilizando el programa NTSYS pc v. 2.1 (Rohlf, 2000), calculando una matriz de distancia mediante el coeficiente SM (Simple Matching Coefficient) y empleando como método de agrupamiento jerárquico UPGMA, a fin de determinar la similitud entre las especies y comprobar si estos caracteres utilizados son consistentes con el tratamiento taxonómico propuesto para Bursera. La matriz de datos se basó en nueve rasgos de la epidermis foliar (Cuadro 1) codificados como multiestado, ocho de ellos cualitativos y uno cuantitativo (índice estomático). El carácter cuantitativo fue codificado como cualitativo utilizando un método de sub-grupos homogéneos (Simon, 1983; Rae, 1998) basado en una prueba de Duncan realizada en SPSS v. 13.0. Los análisis se realizaron considerando como unidades taxonómicas las especies, debido a que los caracteres fueron homogéneos entre los individuos estudiados de cada taxon. En el fenograma obtenido se hace referencia a la línea fenón, es decir el límite trazado a un determinado valor del coeficiente de similitud, para definir un agrupamiento de interés taxonómico.

\section{RESULTADOS}

Epidermis foliar

Todas las especies estudiadas presentan células de forma poligonal y paredes rectas (Fig. 1; Fig. 4), las cuales son más gruesas y con numerosas punteaduras en $B$. glabra, $B$. graveolens y $B$. inversa en comparación con las especies restantes. En todos los casos las hojas son hipostomáticas, con estomas anomocíticos (Fig. 2), a excepción de $B$. glabra que presenta estomas del tipo ciclocítico y anomocítico (Fig. 2a). El índice estomático, tal como se aprecia en el Cuadro 2, separa al conjunto estudiado en tres grupos estadísticamente distintos. 
Cuadro 1. Matriz de caracteres y estado de caracteres de la epidermis foliar para el análisis de agrupamiento en especies de Bursera.

\begin{tabular}{ll}
\hline Carácter & Codificación \\
\hline Relieve del contorno celular & Acanalado (0); Levantado (1) \\
$\begin{array}{l}\text { Curvatura de las paredes periclinales } \\
\text { externas }\end{array}$ & Plana (0); Convexa (1); Cóncava (2) \\
Relieve fino de la pared celular & Lisa (0); Estriada (1) \\
Tricomas & Ausente (0); Presente (1) \\
Forma de los tricomas & Globular (0); En forma de mazo (1) \\
Ornamentación de los tricomas & Verrucosa (0); Verrucosa-baculada (1); \\
& Baculada (2) \\
Posición de los estomas & A nivel (0); Levantados (1); Hundidos (2) \\
Índice estomático* & a (0); b (1); c (3) \\
\hline
\end{tabular}

* Codificado como cualitativo

Se observan tricomas tectores y glandulares en cinco especies (Fig. 3), mientras que $B$. karsteniana es totalmente glabra. Los pelos tectores son multicelulares y uniseriados, con una cutícula gruesa y mayor densidad en la superficie abaxial, excepto para $B$. tomentosa, donde la densidad de tricomas es alta en ambas superficies. Los tricomas glandulares presentan un pie corto y una cabeza globosa multicelular en casi todas las especies, mientras que en $B$. inversa éstos tienen forma de mazo (Fig. 6b).

Micromorfología de la epidermis foliar

En el Cuadro 2 se presentan las características más relevantes de la micromorfología foliar de las especies estudiadas. El margen de la pared anticlinal está levantado en $B$. simaruba (Figura 4e), mientras que en las demás se observa hundido. La pared periclinal externa es convexa en la mayoría, pero plana en B. glabra y curva hacia adentro en $B$. simaruba. El patrón de ornamentación de la superficie de las células epidérmicas es estriado en cuatro especies y liso en $B$. karsteniana y $B$. simaruba (Figs. 4d, 4e). Las ceras epicuticulares son escasas en ambas superficies y con forma laminar o de cristaloides granulosos.

Los estomas están ligeramente hundidos en $B$. glabra y $B$. graveolens (Figs. $5 \mathrm{a}, 5 \mathrm{~b}$ ), levantados en $B$. tomentosa (Fig. 5f) y al mismo nivel de las células epidérmicas en B. karsteniana y B. simaruba (Figs. 5c, 5d, 5e). Tal como se aprecia en la 
Cuadro 2. Características de la epidermis foliar en especies de Bursera.

\begin{tabular}{|c|c|c|c|c|c|c|}
\hline Carácter & B. glabra & B. graveolens & B. tomentosa & B. karsteniana & B. inversa & B. simaruba \\
\hline $\begin{array}{l}\text { Forma de las } \\
\text { células }\end{array}$ & poligonal & poligonal & poligonal & poligonal & poligonal & poligonal \\
\hline $\begin{array}{l}\text { Relieve del } \\
\text { contorno celular }\end{array}$ & $\begin{array}{l}\text { ligeramente } \\
\text { acanalado }\end{array}$ & acanalado & acanalado & acanalado & acanalado & acanalado \\
\hline $\begin{array}{l}\text { Forma de } \\
\text { las paredes } \\
\text { anticlinales }\end{array}$ & recta & recta & recta & recta & recta & recta \\
\hline $\begin{array}{l}\text { Curvatura de la } \\
\text { pared periclinal } \\
\text { externa de las } \\
\text { células }\end{array}$ & aplanada & $\begin{array}{l}\text { ligeramente } \\
\text { convexa }\end{array}$ & convexa & $\begin{array}{l}\text { ligeramente } \\
\text { convexa }\end{array}$ & $\begin{array}{l}\text { ligeramente } \\
\text { convexa }\end{array}$ & cóncava \\
\hline $\begin{array}{l}\text { Relieve fino de } \\
\text { la pared celular } \\
\text { (ornamentación } \\
\text { secundaria) }\end{array}$ & $\begin{array}{l}\text { ligeramente } \\
\text { estriada }\end{array}$ & estriada & estriada & lisa & estriada & lisa \\
\hline $\begin{array}{l}\text { Ornamentación } \\
\text { de los tricomas }\end{array}$ & $\begin{array}{l}\text { ligeramente } \\
\text { verrucosa }\end{array}$ & $\begin{array}{l}\text { ligeramente } \\
\text { verrucosa }\end{array}$ & $\begin{array}{l}\text { verrucosa- } \\
\text { baculada }\end{array}$ & glabra & $\begin{array}{l}\text { cortamente } \\
\text { baculada }\end{array}$ & baculada \\
\hline $\begin{array}{l}\text { Posición de los } \\
\text { estomas }\end{array}$ & $\begin{array}{l}\text { ligeramente } \\
\text { hundidos }\end{array}$ & $\begin{array}{l}\text { ligeramente } \\
\text { hundidos }\end{array}$ & levantados & a nivel & a nivel & a nivel \\
\hline $\begin{array}{l}\text { Índice } \\
\text { estomático }\end{array}$ & $18.35^{b}$ & $11.08^{\mathrm{a}}$ & $25.72^{\mathrm{c}}$ & $27.22^{\mathrm{c}}$ & $17.57^{b}$ & $11.73^{\mathrm{a}}$ \\
\hline
\end{tabular}

figura 6, la ornamentación de la superficie de los tricomas tectores es verrucosa en B. glabra y $B$. graveolens, baculada en $B$. inversa y $B$. simaruba e intermedia entre ambos tipos en $B$. tomentosa.

\section{Análisis fenético}

El fenograma obtenido muestra que las especies forman dos grupos (Fig. 7), uno donde se ubican las representantes del subgénero Bursera (línea fenón ca. 0.41) y el otro que incluye las del subgénero Elaphrium (línea fenón ca. 0.64). En el primero se aprecia que $B$. simaruba se separa considerablemente del subgrupo formado por $B$. karsteniana $+B$. inversa; mientras que para el subgénero Elaphrium se observa que $B$. glabra y $B$. graveolens son bastante similares entre sí y se diferencian de B. tomentosa. 
Castro y Torrecilla: Epidermis foliar en algunas especies de Bursera y sus implicaciones taxonómicas
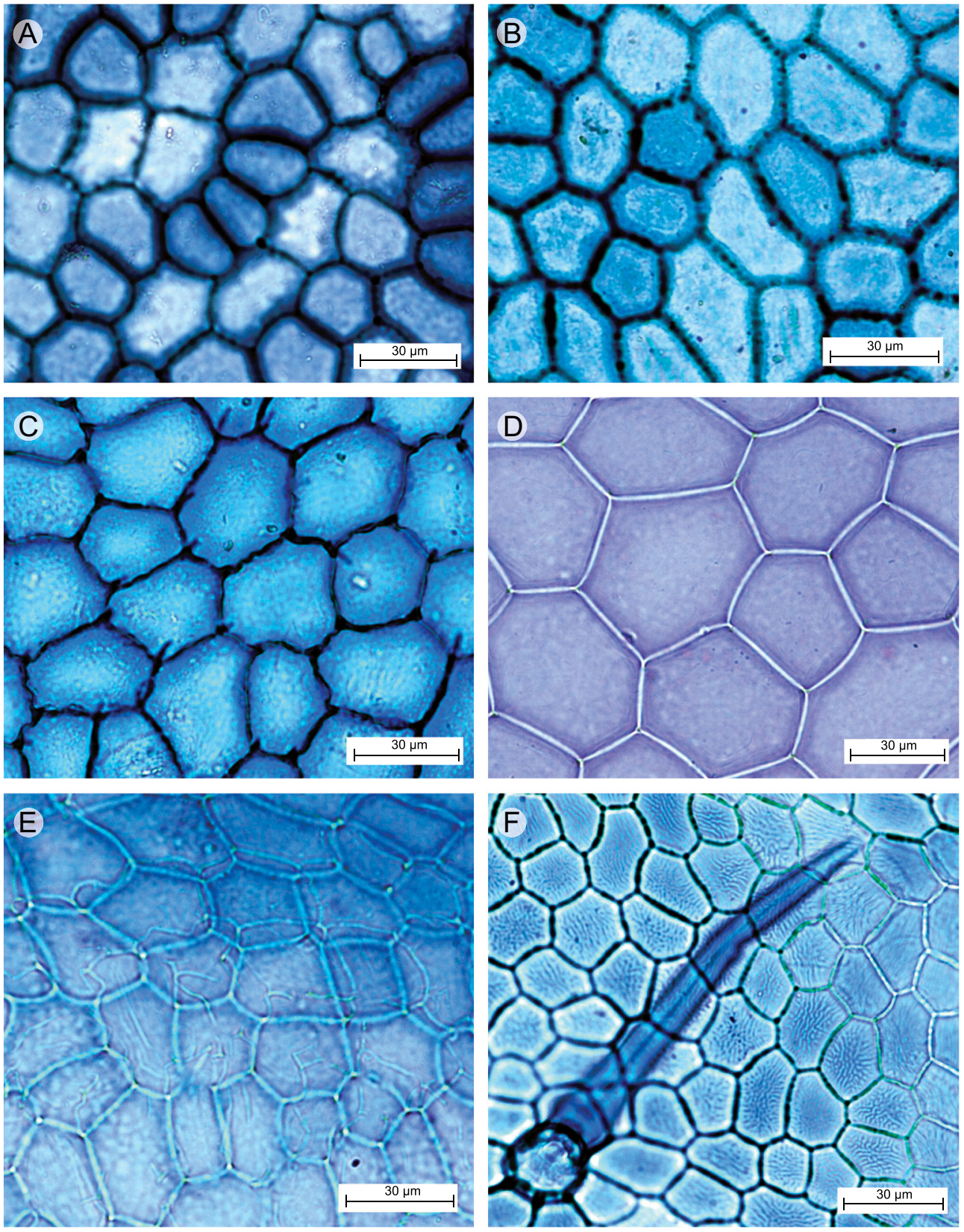

Fig. 1. Vista paradérmica (MO) de la cara adaxial en la lámina foliar de especies de Bursera. A. B. glabra; B. B. graveolens; C. B. inversa; D. B. karsteniana; E. B. simaruba; F. B. tomentosa. 

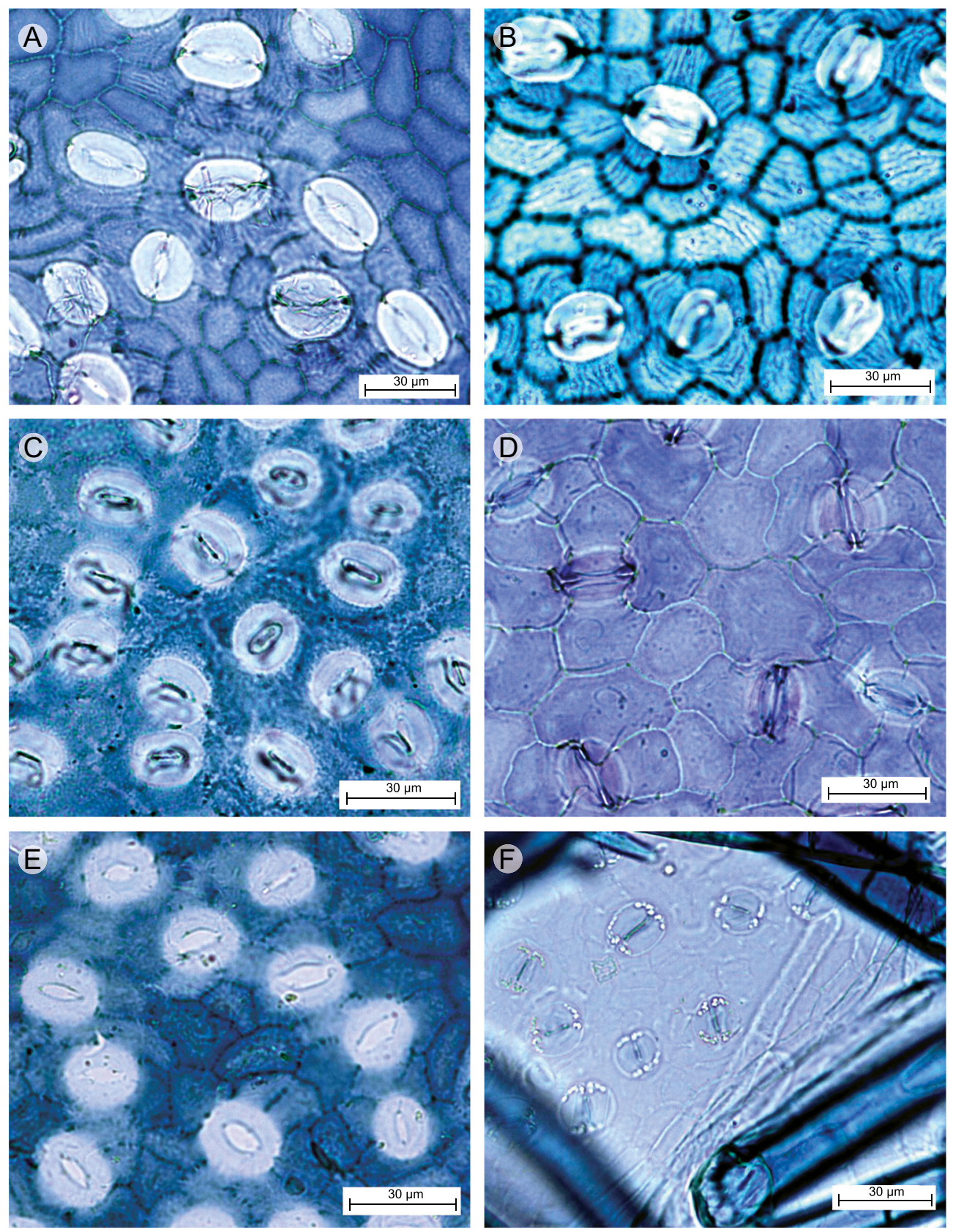

Fig. 2. Vista paradérmica (MO) de la cara abaxial en la lámina foliar de especies de Bursera. A. B. glabra; B. B. graveolens; C. B. inversa; D. B. karsteniana; E. B. simaruba; F. B. tomentosa. 
Castro y Torrecilla: Epidermis foliar en algunas especies de Bursera y sus implicaciones taxonómicas
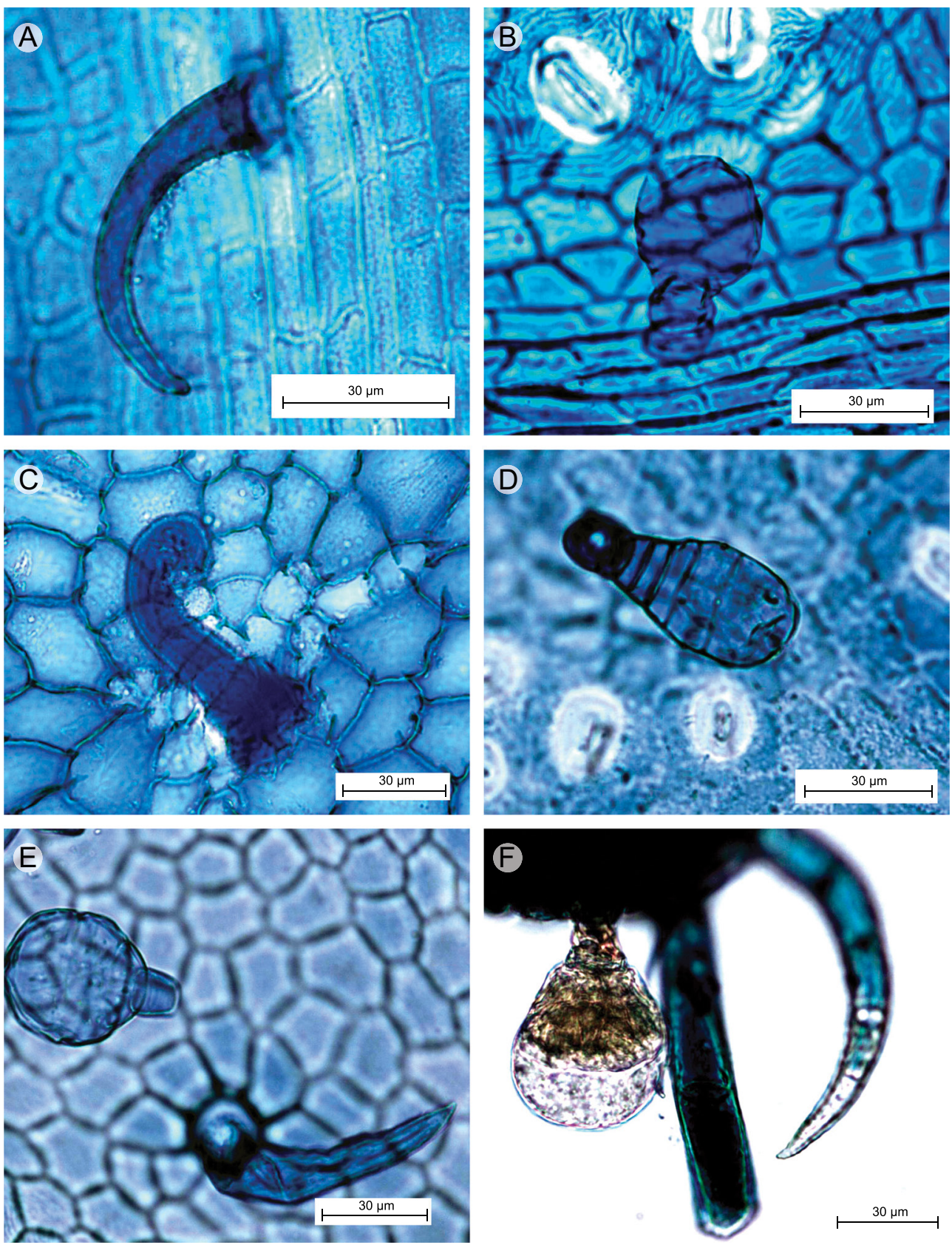

Fig. 3. Tricomas (MO) de especies de Bursera. A. B. glabra; B. B. graveolens; C. B. inversa; D. B. simaruba; E-F. B. tomentosa. 

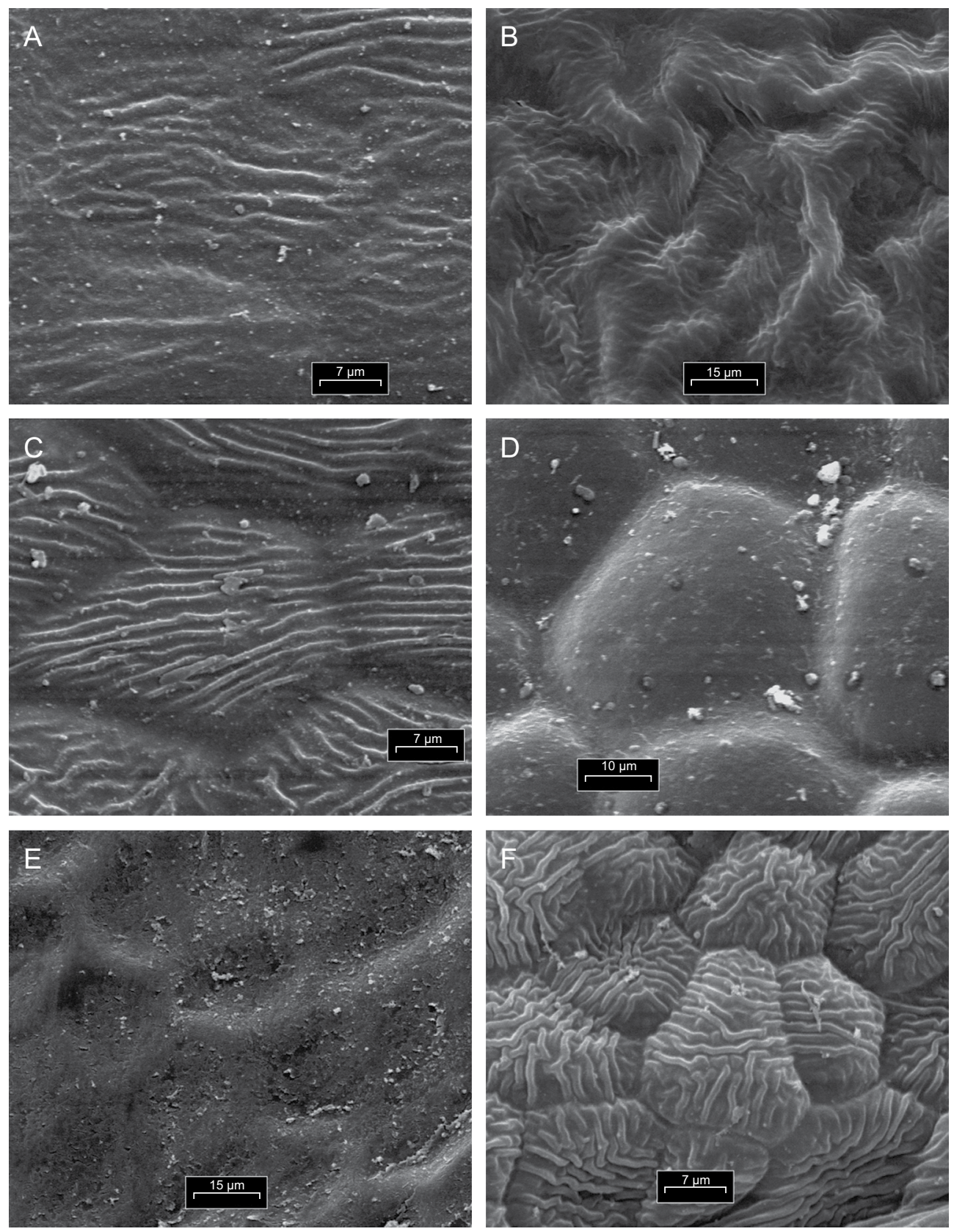

Fig. 4. Vista paradérmica (MEB) de la cara adaxial en la lámina foliar de especies de Bursera. A. B. glabra; B. B. graveolens; C. B. inversa; D. B. karsteniana; E. B. simaruba; F. B. tomentosa. 
Castro y Torrecilla: Epidermis foliar en algunas especies de Bursera y sus implicaciones taxonómicas
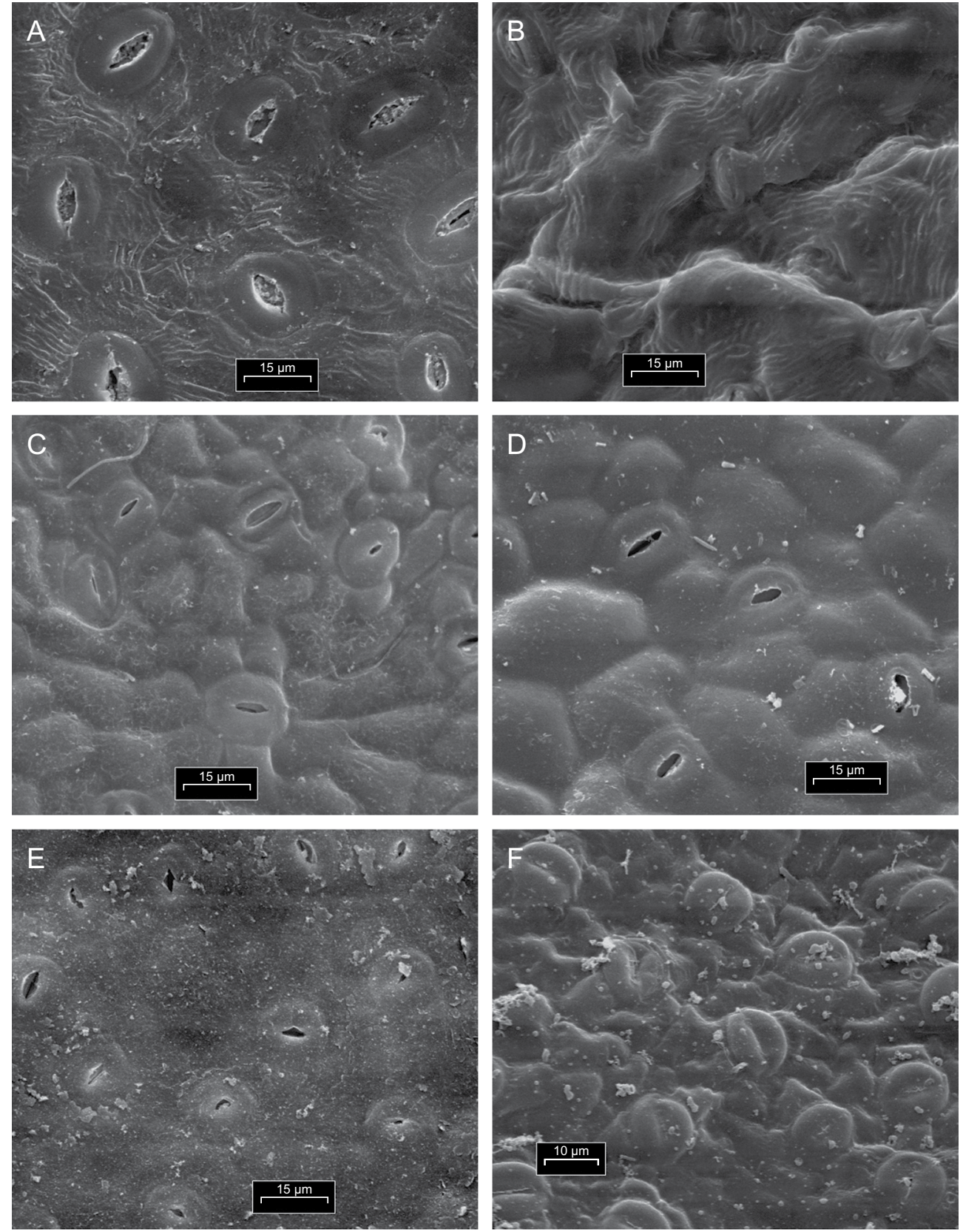

Fig. 5. Vista paradérmica (MEB) de la cara abaxial en la lámina foliar de especies de Bursera. A. B. glabra; B. B. graveolens; C. B. inversa; D. B. karsteniana; E. B. simaruba; F. B. tomentosa. 

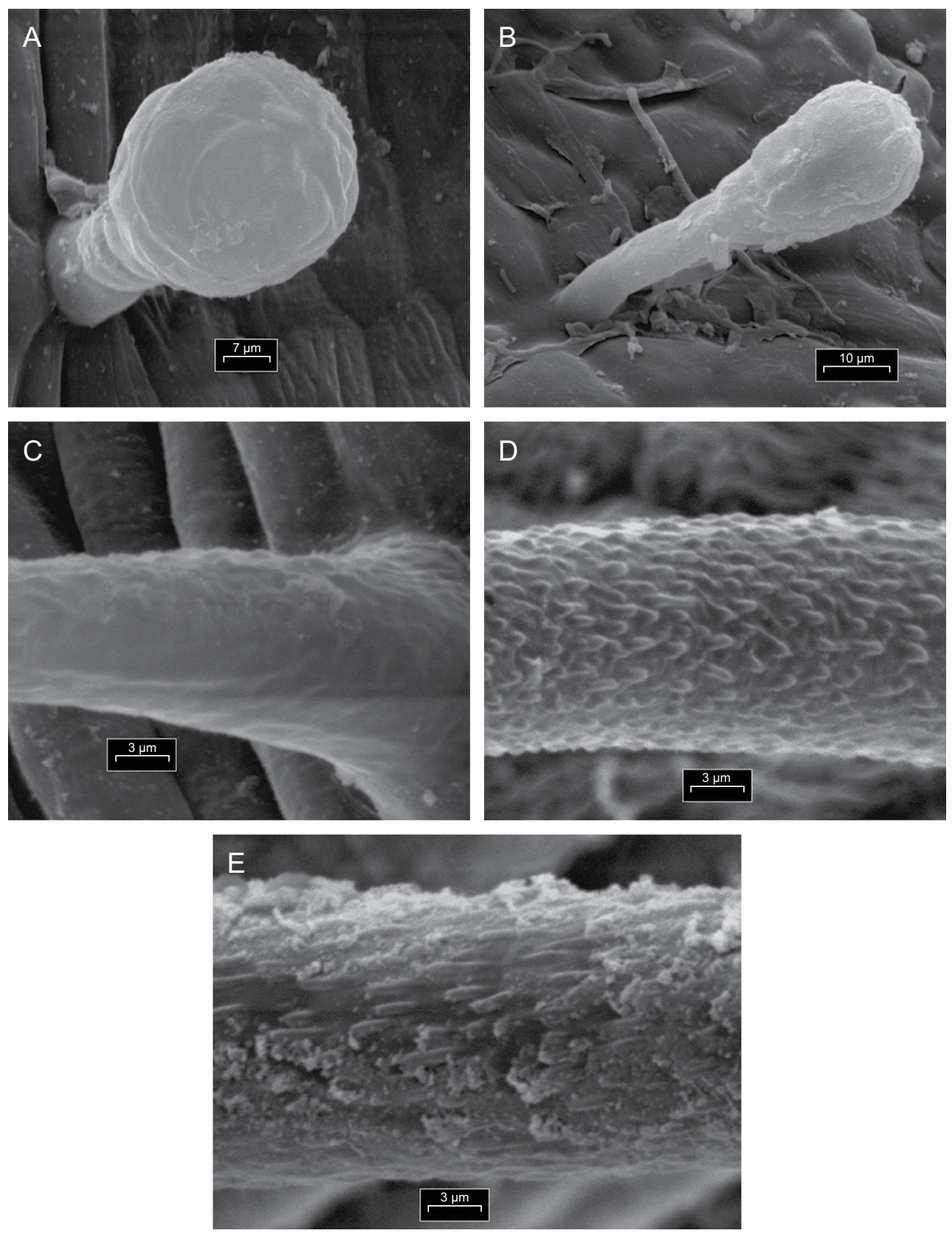

Fig. 6. Tricomas (MEB) de especies de Bursera A. Tricoma glandular de B. glabra; B. Tricoma glandular de B. inversa; C. Tricoma tector de B. glabra; D. Tricoma tector de $B$. tomentosa; E. Tricoma tector de B. simaruba. 
Castro y Torrecilla: Epidermis foliar en algunas especies de Bursera y sus implicaciones taxonómicas

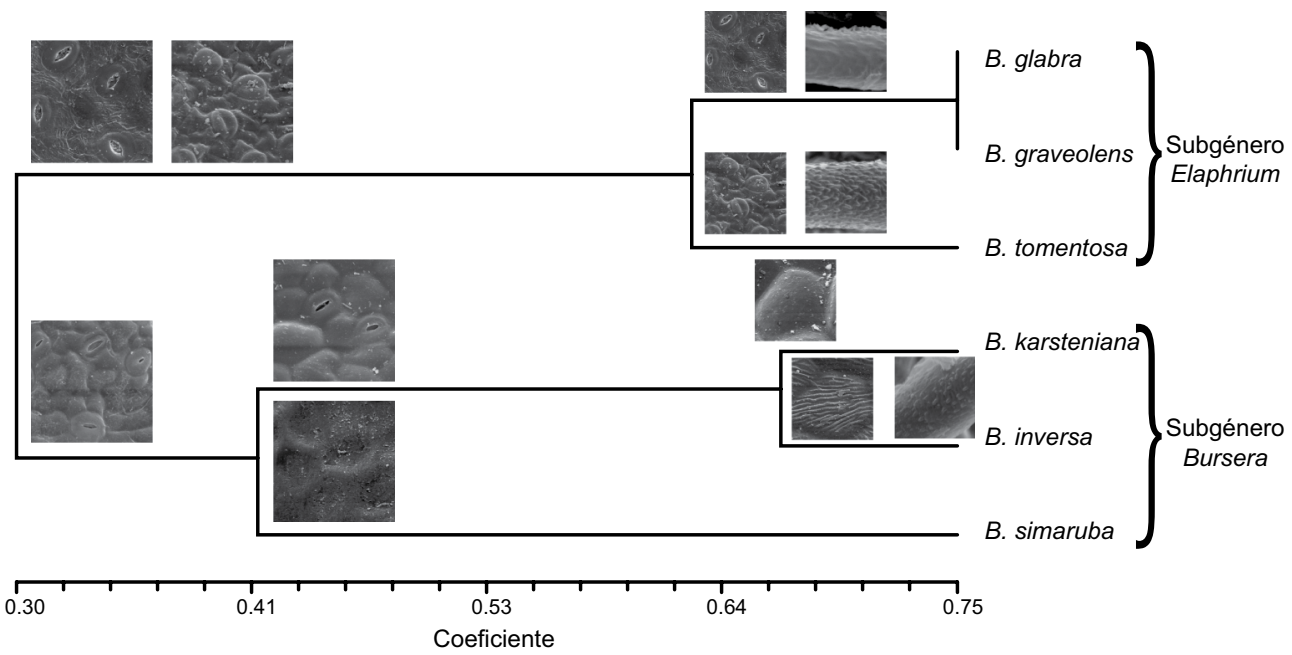

Fig. 7. Análisis de conglomerado con base en la micromorfología de la epidermis foliar en especies de Bursera.

Se presenta una clave que permite diferenciar a las especies estudiadas con base en los caracteres de la epidermis foliar evaluados.

Clave para separar especies de Bursera basada en los caracteres de la epidermis foliar

1 Estomas ubicados a nivel de las células epidérmicas normales

2 (subgénero Bursera)

1 Estomas ubicados por debajo o por encima del nivel de las células epidérmicas normales 4 (subgénero Elaphrium)

2 Células epidérmicas con el relieve del contorno celular levantado y curvatura de la pared periclinal externa cóncava B. simaruba

2 Células epidérmicas con el relieve del contorno celular acanalado y curvatura de la pared periclinal externa convexa

3 Hojas glabras y relieve de la pared externa liso B. karsteniana

3 Hojas con tricomas tectores cortamente baculados, y relieve fino de la pared externa estriado.

B. inversa

4 Estomas ubicados por encima del nivel de las células epidérmicas normales, tricomas tectores verrucoso-baculados B. tomentosa 
4 Estomas ubicados por debajo del nivel de las células epidérmicas normales, tricomas tectores baculados. ............................................ 5

5 Curvatura de la pared periclinal externa plana, índice estomático $\geq 15$ B. glabra

5 Curvatura de la pared periclinal externa convexa, índice estomático $<15$ B. graveolens

\section{DISCUSIÓN}

Las especies estudiadas son hipostomáticas, con estomas principalmente del tipo anomocítico y tricomas similares, características que, como se ha señalado, son comunes al género Bursera (Metcalfe y Chalk, 1950).

Se ha mostrado que el índice estomático tiene valor taxonómico (Solereder, 1908), habiéndose evaluado en diversos grupos de plantas en los que se ha puesto de manifiesto que contribuye a la identificación de las especies (Kadiri y Olowokudejo, 2008; Szymura y Wolski, 2011; Thakur y Patil, 2011). En este estudio, el índice estomático presenta valores distintos para todas las especies evaluadas, sin embargo los grupos formados mediante análisis estadístico no son congruentes con la delimitación de los subgéneros en este grupo (McVaugh y Rzedowski, 1965; Becerra y Venable, 1999; Becerra, 2003), lo cual podría indicar que para Bursera el índice estomático no contribuye en la subdivisión del género.

En relación con la micromorfología de la epidermis foliar, todas las especies estudiadas presentan paredes anticlinales rectas, lo cual probablemente sea una característica común a Bursera y otros grupos estrechamente relacionados como Commiphora (observ. personal). En este sentido, se ha indicado que el borde de las paredes anticlinales de las células pueden tener una alta significancia taxonómica (Barthlott, 1984); no obstante, es importante evaluar tal carácter en un mayor número de especies de Bursera y géneros relacionados a fin de establecer su constancia en este grupo.

Los análisis fenéticos muestran que los caracteres de la epidermis foliar evaluados agrupan a las especies estudiadas en los dos subgéneros propuestos para Bursera (Bullock, 1936; McVaugh y Rzedowski, 1965; Gillet, 1980; Toledo-Manzur, 1982; Becerra y Venable, 1999). Las especies del subgénero Bursera presentan los estomas al nivel de las células epidérmicas típicas mientras que las de Elaphrium las tienen a una altura distinta. No obstante, a fin de establecer la constancia de este carácter se requiere evaluar un mayor número de especies de ambos taxones. 
Se discrimina un grupo que encierra a las especies del subgénero Elaphrium (B. glabra, B. graveolens y B. tomentosa), las cuales presentan estomas a un nivel distinto que las células epidérmicas típicas (hundidos o levantados) y ornamentación secundaria de la pared celular estriada. Estos resultados apoyan los estudios taxonómicos de este grupo que indican que las especies mencionadas están estrechamente relacionadas entre sí (McVaugh y Rzedowski, 1965; Becerra y Venable, 1999). Dentro del subgénero se aprecia que $B$. glabra y $B$. graveolens son bastante afines, compartiendo la presencia de estomas ligeramente hundidos y la ornamentación de los tricomas tectores es verrucosa, mientras que $B$. tomentosa se diferencia de las dos especies anteriores por presentar los estomas levantados y tricomas tectores con una ornamentación verrucosa-baculada.

Las especies del subgénero Elaphrium se han separado en dos conjuntos con base en sus rasgos morfológicos, caracterizándose el grupo copallifera por presentar los sépalos libres y el pireno cubierto por el pseudoarilo en dos terceras partes o más, mientras que en el de glabrifolia los sépalos son unidos y el pseudoarilo cubre menos de los dos tercios del pireno (Toledo-Manzur, 1982). La existencia de estos dos grupos dentro de Elaphrium es apoyada por algunos análisis filogenéticos (Becerra y Venable, 1999; Becerra, 2003). Espinosa et al. (2006) indican que B. glabra y $B$. graveolens estarían dentro del grupo glabrifolia y $B$. tomentosa en el grupo copallifera con base en sus características morfológicas. No obstante, De Nova et al. (2012) realizan una reconstrucción filogenética de Bursera basada en datos moleculares, en la que se incluye a estas tres especies, quedando B. glabra en el grupo copallife$r a$, mientras que $B$. graveolens y $B$. tomentosa se ubican en el conjunto glabrifolia. Los agrupamientos obtenidos en este estudio con base en la micromorfología foliar apoyan la propuesta morfológica de ubicación de las especies, pero no la molecular, por lo que se debe estudiar un mayor número de taxones para determinar su posible utilidad sistemática.

Las especies consideradas del subgénero Bursera han sido incluidas dentro del complejo B. simaruba por sus similitudes morfológicas (Daly, 1993; Espinosa et al., 2006). No obstante, el fenograma muestra que con respecto a la micromorfología foliar, $B$. karsteniana y $B$. inversa son más parecidas entre sí que con respecto a $B$. simaruba, debido a que esta última presenta los bordes de las células levantados y la pared periclinal externa es cóncava, mientras que $B$. karsteniana + $B$. inversa tienen los bordes de las paredes celulares acanalados y la pared periclinal externa es convexa. Por su parte, es posible diferenciar a $B$. karsteniana debido a que la ornamentación de la pared celular es lisa mientras que en $B$. inversa es estriada. 
Estudios moleculares recientes en los que se incluye a $B$. inversa sugieren que esta especie no forma parte de ninguno de los dos subgéneros en que tradicionalmente ha sido dividido Bursera (Martínez-Habibe, 2012); adicionalmente la morfología de los cotiledones es distinta a lo señalado para el género (observ. personal), lo cual indica que la posición taxonómica de $B$. inversa no está clara. En el caso de B. karsteniana, Martínez-Habibe (2012) incluye en sus análisis filogenéticos a esta planta bajo el sinónimo $B$. trinitensis, quedando ubicada en un grupo hermano al complejo simaruba dentro del cual se encuentra la propia $B$. simaruba.

Con base en lo anterior, se aprecia que los caracteres de la epidermis foliar separan bien entre sí a estas tres especies, pero no parecen aportar información para resolver la posición sistemática de las mismas.

Los resultados obtenidos indican que los rasgos estudiados pueden tener un alto valor taxonómico en Bursera, pero se requiere continuar con los estudios en el grupo a fin de esclarecerlo.

\section{AGRADECIMIENTOS}

Los autores agradecen al Consejo de Desarrollo Científico y Humanístico de la Universidad Central de Venezuela por el financiamiento de la presente investigación.

\section{LITERATURA CITADA}

Andrés-Hernández, R. y D. Espinosa-Organista. 2002. Morfología de plántulas de Bursera Jacq. ex L. y sus implicaciones filogenéticas. Bol. Soc. Bot. Méx. 70: 5-12.

Barthlott, W. 1981. Epidermal and seed surface characters of plants: systematic applicability and some evolutionary aspects. Nord. J. Bot. 1: 345-355.

Barthlott, W. 1984. Microstructural features of seed surfaces. In: Heywood, V. H. y D. M. Moore (eds.). Current concepts in plant taxonomy. Academic Press. London, UK. pp. 95-105.

Barthlott, W., C. Neinhuis, D. Cutler, F. Disco, I. Meusel, I. Theisen y H. Wilhelmi. 1998. Classification and terminology of epicuticular waxes. Bot. J. Linn. Soc. 126: 237-260.

Becerra, J. 2003. Evolution of Mexican Bursera (Burseraceae) inferred from ITS, ETS and 5S ribosomal DNA sequences. Mol. Phyl. Evol. 26: 300-309.

Becerra, J., K. Noge, S. Olivier y L. Venable. 2012. The monophyly of Bursera and its impacts for divergence times of Burseraceae. Taxon 61: 333-343.

Becerra, J. y L. Venable. 1999. Nuclear ribosomal DNA phylogeny and its implications for evolutionary trends in Mexican Bursera (Burseraceae). Amer. J. Bot. 86: 1047-1057. 
Castro y Torrecilla: Epidermis foliar en algunas especies de Bursera y sus implicaciones taxonómicas

Bullock, A. 1936. Notes on the Mexican species of the genus Bursera. Bull. Misc. Inf. Kew 1936: 346-387.

Daly, D. 1993. Notes on Bursera in South America, including a new species. Studies in Neotropical Burseraceae VII. Brittonia 45: 240-246.

Daly, D. 1997. Burseraceae. In: Berry, P., B. Holst y K. Yatskievych (eds.). Flora of the Venezuelan Guayana. Vol. 3. Timber Press. Portland, USA. pp. 688-728.

Dean, M. y P. Ashton. 2008. Leaf surfaces as a taxonomical tool: the case of Carex section Phacocystis (Cyperaceae) in the British Isles. Plant Syst. Evol. 273: 97-105.

De Nova, A., R.,Medina, J. Montero, A. Weeks, J. Rosell, M. Olso, L. Eguiarte and S. Marañon. 2012. Insights into the historical construction of species-rich Mesoamerican seasonally dry tropical forests: the diversification of Bursera (Burseraceae, Sapindales). New Phytol. 193: 276-287.

Espinosa, D., J. Llorente y J. Morrone. 2006. Historical biogeographical patterns of the species of Bursera (Burseraceae) and their taxonomic implications. J. Biogeogr. 33: 1945-1958.

Gillett, J. B. 1980. Commiphora (Burseraceae) in South America and its relationship to Bursera. Kew Bull. 34: 569-589.

Kadiri, A. B. y J. D. Olowokudejo. 2008. Comparative foliar epidermal morphology of the West African species of the genus Afzelia Smith (Leguminosae-Caesalpinioideae. Gayana Bot. 65: 84-92.

Loza-Cornejo, S. y T. Terrazas. 2003. Epidermal and hypodermal characteristics in North America Cactoideae (Cactaceae). J. Plant Res. 116: 27-35.

Martínez-Habibe, C. 2012. Systematics, biogeography and leaf anatomy and architecture of Bursera subgen. Bursera (Burseracaeae) in the Greater Antilles and the Bahamas. Dissertation, PhD Degree in Botany. Claremont Graduate University. Claremont, USA. 114 pp.

McVaugh, R. y J. Rzedowski. 1965. Synopsis of the genus Bursera L. in western Mexico, with notes on the material of Bursera collected by Sessé \& Mociño. Kew Bull. 18: 317-382.

Metcalfe, C. R. y L. Chalk. 1950. Anatomy of the Dicotyledons. Vol. 1 y 2. Clarendon Press. Oxford, UK. 1500 pp.

Ogunkunle, A. y F. Oladele. 2008. Leaf epidermal studies in some Nigerian species of Ficus L. (Moraceae). Plant Syst. Evol. 274: 209-221.

Rae, T. C. 1998. The logical basis for the use of continuous characters in phylogenetic systematics. Cladistics 14: 221-228.

Rohlf, F. 2000. NTSYS-pc. Numerical taxonomy and multivariate analysis system. Version 2.1. Exeter Software. Applied Biostatistics. New York, USA.

Shuff, T. y J. Thomas. 1993. Normal ontogeny and cool temperature-induced aberrant floral development in Glycine $\max$ (Fabaceae). Amer. J. Bot. 80: 429-448.

Simon, C. 1983. A new coding procedure for morphometric data with an example from periodical cicada wing veins. In: Felsenstein, J. (ed.). Numerical taxonomy. SpringerVerlag. Berlin, Alemania. pp. 378-382.

Solereder, H. 1908. Systematic anatomy of the dicotyledons. Clarendon Press. Oxford, UK. $1182 \mathrm{pp}$. 
Srivastava, A. 1978. Study of leaf epidermis in the genus Digitaria Rich. (Gramineae). J. Indian Bot. Soc. 57: 155-160.

Stace, C. 1965. Cuticular studies as an aid to plant taxonomy. Bull. Br. Mus. Nat. Hist. Bot. 4: 3-78.

Stace, C. 1980. The significance of the leaf epidermis in the taxonomy of the Combretaceae: conclusions. Bot. J. Linn. Soc. 81: 327-339

Stace, C. 1984. The taxonomic importance of the leaf surface. In: Heywood, V. H. y D. M. Moore (eds.). Current concepts in plant taxonomy. Academic Press. London, UK. pp. 67-94.

Szymura, M. y K. Wolski. 2011. Leaf epidermis traits as tools to identify Solidago L. taxa in Poland. Acta Biol. Cracov. 53: 38-46.

Thakur, H. A. y D. A. Patil. 2011. The foliar epidermal studies in some hitherto unstudied Euphorbiaceae. Curr. Bot. 2: 22-30.

Toledo-Manzur, C. 1982. El género Bursera (Burseraceae) en Guerrero (México). Tesis de Licenciatura. Facultad de Ciencias, Universidad Nacional Autónoma de México. México, D.F., México. 182 pp.

Yang, H., H. Wang y D. Li. 2008. Comparative morphology of the foliage leaf epidermis with emphasis on papillae characters, in key taxa of woody bamboos of Asian tropics (Poaceae: Bambusoideae). Bot. J. Linn. Soc. 156: 411-423.

Weeks, A., D. Daly y B. Simpson. 2005. The phylogenetic history and biogeography of the frankincense and myrrh family (Burseraceae) based on nuclear and chloroplast sequence data. Mol. Phyl. Evol. 35: 85-101.

Wilkinson, H. 1979. The plant surface (mainly leaf). Stomata. In: Metcalfe, C. y L. Chalk (eds.). Anatomy of dicotyledons. Vol 1. 2a ed. Clarendon Press. Oxford, UK. 113 pp.

Zou, P., J. Liao y D. Zhang. 2008. Leaf epidermal micromorphology of Cercis (Fabaceae: Caesalpinioideae). Bot. J. Linn. Soc. 158: 539-547. 


\section{APÉNDICE}

Listado de especímenes utilizados en el estudio de la epidermis foliar del género Bursera Jacq. ex L. en Venezuela.

Bursera glabra (Jacq.) Triana \& Planch.

Castro, M. y J. Guevara 153 (MY), estado Mérida; Castro, M. y J. Guevara 154 (MY), estado Mérida; Trujillo, B. 6418 (MY), estado Lara.

Bursera graveolens (Kunth) Triana \& Planch.

Bernardi 508 (MERF), estado Mérida; Castro, M. y J. Guevara 161-A (MY), estado Mérida; Ruíz, T. y col. 4899-452 (MY), estado Trujillo.

Bursera karsteniana Engl.

Cárdenas, L. 1708 (VEN), estado Falcón; Castro, M. s/n (MY), estado Aragua; Trujillo, B. 14815 (MY), estado Aragua.

Bursera inversa Daly

Castro, M. y col. 155 (MY), estado Zulia.

Bursera simaruba (L.) Sarg.

Castro, M. y D. Conde 106 (MY), estado Aragua; Castro, M. y col. 156 (MY), estado Mérida; Monzón, F. s/n (MY), estado Aragua.

Bursera tomentosa (Jacq.) Triana \& Planch.

Alvarado, H. y col. 266 (MY), estado Lara; Castro, M. y D. Jáuregui 152 (MY), estado Aragua; Granada, W. y B. Medina 08 (MY), estado Aragua. 